Pacific Journal of Mathematics

NORMAL BASES FOR QUADRATIC EXTENSIONS 


\section{NORMAL BASES FOR QUADRATIC EXTENSIONS}

\section{Charles SMALL}

This note complements the author's paper in Journal of Pure and Applied Algebra, 2 (1972), in which a computation is made of the functor which associates to each commutative ring $k$ its group $Q(k)$ of quadratic extensions, where "quadratic extension of $k$ " means "Galois extension of $k$ with respect to a group of order two". In general, quadratic extensions are rank two projective $k$-modules; the free ones form a subgroup $Q_{F}(k)$ of $Q(k)$. Among the free ones are some which admit a normal basis (definition recalled below); these form a subgroup $Q_{N B}(k)$. This paper studies the filtration $0 \subseteq Q_{N B} \subseteq Q_{F} \subseteq Q$.

The starting point for the computation in [5] was the construction of a functor $\mathscr{R}$ and a natural monomorphism $\beta: \mathscr{R}(k) \rightarrow Q(k)$ (definitions recalled below). Our first observation here is that $\beta$ is an isomorphism $\mathscr{R}(k) \rightarrow Q_{F}(k)$ and that the subfunctor $R$ of $\mathscr{R}$ which corresponds to $Q_{N B}$ (via $\beta$ ) is one studied by Micali and Villamayor in [3]. These results, which follow without difficulty from the work in [5], allow us to find simple necessary and sufficient conditions for $Q_{N B}(k)=Q_{F}(k)$, and at the other extreme to produce an infinite family of $k$ for which $0=Q_{N B}(k) \neq Q_{F}(k)$.

Now it is known that $Q_{N B}$ is isomorphic to the Harrison cohomology functor $H^{2}(, \Pi)$ where $\Pi$ is the group of order two. (See [2] and [4] for the following more general result: The group of normalbasis extensions of $k$ with Galois group $G$ is naturally isomorphic to $H^{2}(k, G)$ for any abelian group $G$.) In $\S 2$ we establish directly, by a series of simple calculations, an isomorphism $\alpha: H^{2}(, \Pi) \rightarrow R$. (In fact $\beta \alpha$ turns out to be the isomorphism $H^{2}(, \Pi) \rightarrow Q_{N B}$ of [2] and [4].) This provides a new proof of the isomorphism $H^{2}(, \Pi)=Q_{N B}$ and also, in our opinion, sheds new light on it by identifying the functor in question with that of Micali-Villamayor. The isomorphism $Q_{N B}=H^{2}(, \Pi)$ generalizes nicely, as indicated above; on the other hand, for quadratic extensions the description in terms of Harrison cohomology is unnecessarily complicated and $R$ is considerably easier to compute with.

Thanks are due to L. N. Childs and M. Orzech for (respectively) raising and discussing the question.

1. Identification of $R \subseteq \mathscr{R}$ with $Q_{N B} \leqq Q_{F}$. Throughout, $k$ is an arbitrary commutative ring (with 1 ) and $\Pi$ is the group of order two. We will associate various groups with $k$, using the same symbol 
* for the operation in each; our results relate the groups in such a way that, among other things, this ambiguity of notation is rendered harmless.

By a quadratic extension of $k$ we mean a (commutative) $k$-algebra which is a Galois extension of $k$ with respect to $\Pi$, in the sense of [1]. If $A$ and $B$ are quadratic extensions of $k$ then so is $A * B$, the subring of elements of $A \bigotimes_{k} B$ left fixed by $\sigma_{A} \otimes \sigma_{B}$ (where $\sigma_{A}$ generates the Galois group of $A / k$, etc.). Indeed, * makes the set of isomorphism classes of quadratic extensions of $k$ into an abelian group of exponent $\leqq 2$ (see [5]). This group we denote $Q(k) . \quad Q$ is a functor: $k \rightarrow K$ induces $Q(k) \rightarrow Q(K)$ by $A \mapsto A \bigotimes_{k} K$.

In general, quadratic extensions of $k$ are projective of rank two as $k$-modules ([1], Lemma 4.1). The free ones form a subgroup $Q_{F}(k)$ of $Q(k)$. Among the free quadratic extensions are some which admit a normal basis, that is, a basis of the form $\{w, \sigma w\}$ where $\sigma$ generates the Galois group. These form a subgroup $Q_{N B}(k)$ of $Q_{F}(k)$.

We now recall the construction of the groups $\mathscr{R}(k)$ and $R(k)$, referring to [5] for the proofs. Let $U(k)$ denote the multiplicative group of units of $k$. If $f: R \rightarrow k$ is a homomorphism from a commutative ring $R$ to $k$ and we fix an element $y \in R$, the set

$$
k_{y}=\{x \in k \mid(1-f(y) x) \in U(k)\}
$$

becomes an abelian group under the operation $x_{1} * x_{2}=x_{1}+x_{2}-f(y) x_{1} x_{2}{ }^{1}$. In particular we get a group $k_{n}$ for each $n \in \boldsymbol{Z}$ from the unique homomorphism $\boldsymbol{Z} \rightarrow k$. Write $*$, or $*_{n}$ where necessary, for the group operation in $k_{n}$.

Proposition 1. $\psi(x)=x(1-x)$ defines a natural homomorphism $\psi: k_{2} \rightarrow k_{4}$ whose kernel is the group $I(k)$ of idempotents of $k$.

Proof. We have first to show that $x \in k_{2}$ implies $x(1-x) \in k_{4}$ and that $\psi\left(x_{1} *_{2} x_{2}\right)=\left(\psi x_{1}\right) *_{4}\left(\psi x_{2}\right)$. Both are trivial. The statement about the kernel just says $x(1-x)=0 \Leftrightarrow x=x^{2}$.

Now define $R(k)=$ coker $(\psi)$, so that the sequence

$$
0 \longrightarrow I(k) \longrightarrow k_{2} \stackrel{\psi}{\longrightarrow} k_{4} \longrightarrow R(k) \longrightarrow 0
$$

is exact. Note that $x \in k_{4}$ implies that $x *_{4} x=2 x(1-2 x)$ is in $\psi\left(k_{2}\right)$. This shows that $R(k)$, with the operation $*$ induced by $*_{4}$, is a group of exponent $\leqq 2$. The functor $R$ was first considered in [3, §7], where it is called $G$.

To construct $\mathscr{R}(k)$ we consider first the set $\mathscr{T}(k)$ of triples

1 The reader will have no trouble completing the definition to make $k \mapsto k_{y}$ a functor. 
$(u, a, x)$ where $u \in U(k)$ and $a, x \in k$ satisfy $a^{2} u+4 x=1$. If $(u, a, x)$ and $\left(u^{\prime}, a^{\prime}, x^{\prime}\right)$ are in $\mathscr{T}(k)$ then so is $(u, a, x) *\left(u^{\prime}, a^{\prime}, x^{\prime}\right)=\left(u u^{\prime}, a a^{\prime}\right.$, $\left.x+x^{\prime}-4 x x^{\prime}\right)$, and $*$ is commutative and associative and has $(1,1,0)$ as neutral element. Define " $(u, a, x) \sim\left(u^{\prime}, a^{\prime}, x^{\prime}\right)$ by $v, b$ " to mean: $v \in U(k), b \in k, u^{\prime}=v^{2} u, a^{\prime} v=a-2 b, x^{\prime}=x+b(a-b) u$. Write $(u, a, x) \sim$ $\left(u^{\prime}, a^{\prime}, x^{\prime}\right)$ iff $(u, a, x) \sim\left(u^{\prime}, a^{\prime}, x^{\prime}\right)$ by $v, b$ for some $v, b \in k$. Then $\sim$ is an equivalence relation on $\mathscr{T}(k)$, and is compatible with $*$. (Again, for complete proofs see [5].) Hence $*$ induces an operation, again denoted *, on the set $\mathscr{T}(k) / \sim$ of equivalence classes. In fact $\mathscr{T}(k) / \sim$ with this operation is a group of exponent $\leqq 2$, since $(1,1,0) \sim\left(u^{2}, a^{2}, 2 x-4 x^{2}\right)$ by $v=u, b=2 x$, for any $(u, a, x) \in \mathscr{T}(k)$. This group we call $\mathscr{R}(k)$. $\mathscr{R}$ is, in the obvious way, a functor.

Proposition 2. The map from $k_{4}$ to $\mathscr{T}(k)$ given by $x \mapsto(1-4 x, 1, x)$ induces a natural injective homomorphism $R(k) \rightarrow \mathscr{R}(k)$.

Proof. Immediate from the definitions.

We will identify $R(k)$ with its image in $\mathscr{R}(k)$; thus an element of $\mathscr{R}(k)$ is in $R(k)$ iff it has a representative $(u, a, x)$ with $a=1$. It should be noted that when $2 \in U(k), R(k)=\mathscr{R}(k) \cong U(k) / U(k)^{2}$, and when $k$ has characteristic two, $R(k)=\mathscr{R}(k) \cong k^{+} / \mathscr{P}\left(k^{+}\right)$, where $k^{+}$ is the additive group of $k$ and $\mathscr{P}: k^{+} \rightarrow k^{+}$is the homomorphism $\mathscr{P}(x)=x^{2}+x$. See example (1) below for the equality of $R$ and $\mathscr{R}$ in these extreme cases, and see [5] for the identification with the group of square classes (resp. $\mathscr{P}$-classes) of $k$.

Now, given $(u, a, x) \in \mathscr{T}(k)$, let $k\{u, a, x\}$ denote a free $k$-module $k s \oplus k t$ with elements $l, s t, t s, s^{2}, t^{2}, \sigma s, \sigma t$ defined by

$$
(*)\left\{\begin{array}{l}
l=a s+2 t \\
s t=t s=2 x s-a u t \\
s^{2}=u l \\
t^{2}=t-x l \\
\sigma s=-s \\
\sigma t=l-t .
\end{array}\right.
$$

THEOREM 3. The first four equations of $(*)$ (extended linearly) give $k\{u, a, x\}$ a well-defined structure of k-algebra with $l=1$, whose isomorphism class depends only on the class of $(u, a, x)$ in $\mathscr{R}(k)$. The map $\sigma$ given by the remaining two equations (extended linearly) is an algebra automorphism of order two, and $k\{u, a, x\}$ is a quadratic extension of $k$ with Galois group generated by $\sigma$. The map $\beta: \mathscr{R}(k) \rightarrow Q(k)$ induced in this way is an injective homomorphism, natural in $k$. The image of $\beta$ is precisely $Q_{F}(k)$; the image of the restriction of $\beta$ to $R(k)$ is precisely $Q_{N B}(k)$. 
REMARK. When $2 \in U(k)$, any $(u, a, x) \in \mathscr{T}(k)$ is equivalent to $\left(u^{\prime}, 1, x^{\prime}\right)$ with $u^{\prime}=1-4 x^{\prime}$ (see below, Example (1)) and $k\left\{u^{\prime}, 1, x^{\prime}\right\}$ is just $k[X] /\left(X^{2}-u^{\prime}\right)$ with the expected Galois automorphism " $\sigma(X)=$ $-X$ ". When $k$ has characteristic two, any $(u, a, x) \in \mathscr{T}(k)$ is equivalent to $\left(1,1, x^{\prime}\right)$ (again, see Example (1) below) and $k\left\{1,1, x^{\prime}\right\}$ is $k[X] /\left(X^{2}+X+x^{\prime}\right)$ with the expected Galois automorphism " $\sigma(X)=$ $X+1$ ". See [5] for the proofs.

Proof. For everything except the last sentence, and for a basisfree description of $k\{u, a, x\}$, we refer to [5, Theorem 2]. If $A$ is a quadratic extension of $k$, the $k$-linear trace map tr: $A \rightarrow k$ given by $\operatorname{tr}(x)=\sigma x+x$ is onto [1, Lemma 1.6] and therefore splits, so that, as $k$-modules, $A=k \oplus M$ for some rank one projective, viz. $M=\operatorname{ker}(\operatorname{tr})$. Now $A$ is free if and only if $M$ is free, for $M=\Lambda_{k}^{2}(A)$. On the other hand, Theorem 3 of [5] shows that $M$ is free if and only if $A$ is in the image of $\beta$. Hence $\beta$ is an isomorphism $\mathscr{R}(k) \rightarrow Q_{F}(k)$ as claimed. ${ }^{2}$

To see that $\beta$ restricts to an isomorphism $R(k) \rightarrow Q_{N B}(k)$, suppose first that the quadratic extension $A$ is in $\beta(R(k))$. According to the first part of the theorem, $A$ has a $k$-basis $\{s, t\}$ with $\sigma t=1-t$ and $1=s+2 t$. But then clearly $t$ and $\sigma t=s+t$ form a normal basis for $A$. Conversely, suppose that $A=k w \oplus k(\sigma w)$ is a normal-basis quadratic extension. Choose an element $b w+c(\sigma w)$ of trace one; then $1=b \operatorname{tr}(w)+c \operatorname{tr}(\sigma w)=(b+c) \operatorname{tr} w$. Hence $\operatorname{tr}(w)$ is invertible, and we can replace $w$ by $t=(\operatorname{tr} w)^{-1} w$ to get a normal basis $A=k t \oplus k(\sigma t)$ with $\sigma t=1-t$. Now let $s=\sigma t-t$. Then $\sigma s=-s$, and moreover, since the trace of an arbitrary element $b t+c(\sigma t)$ is just $b+c$, we have $k s=\operatorname{ker}(\operatorname{tr})$. Clearly $\{s, t\}$ is a basis, and we have $1=t+$ $\sigma t=s+2 t$. Since $\sigma\left(s^{2}\right)=(\sigma s)^{2}=s^{2}$ we have $s^{2}=u .1$ for some $u \in k$, and $u$ is a unit by [5, Lemma 3]. Similarly, $\sigma$ fixes $t-t^{2}$, so that $t^{2}=t-x .1$ for some $x \in k$. Now solving $x .1=t-t^{2}=(s+t) t$ for $s t$ we find $s t=2 x s+(4 x-1) t$; on the other hand, given an expression $s t=b s+c t(b, c \in k)$, computing the trace of each side shows that $c=-u$. Therefore, $s t=2 x s-u t$ and $u+4 x=1$, and we are done.

Now define $A(k)=\{a \in k \mid \exists b \in k,(a+2 b) \in U(k)\}$ and $B(k)=$ $\left\{a \in k \mid \exists c \in k,\left(a^{2}+4 c\right) \in U(k)\right\}$. Clearly $A(k) \subseteq B(k)$; if $a+2 b$ is a unit so is $(a+2 b)^{2}=a^{2}+4(a+b) b$. As a corollary of the theorem we have

\section{COROLlaRY 4. The following are equivalent:}

(i) $Q_{N B}(k)=Q_{F}(k)$, i.e., every free quadratic extension of $k$ admits a normal basis.

${ }^{2}$ The rule $A \mapsto \operatorname{ker}(\operatorname{tr})$ is a homomorphism $Q(k) \rightarrow \operatorname{Pic}(k)$, and $Q(k) / Q_{F}(k)$ is embedded in this way as a subgroup, usually quite a small one, of the Picard group. See [5, Theorem 4] for the precise statement. 


\section{(ii) $A(k)=B(k)$.}

Proof. (i) is equivalent to $R(k)=\mathscr{R}(k)$, i.e., to the property that every element $(u, a, x)$ of $\mathscr{T}(k)$ be equivalent to one of the form $\left(u^{\prime}, 1, x^{\prime}\right)$. It is immediate from the definition of equivalence $(\sim)$ in $\mathscr{T}(k)$ than this is in turn equivalent to (ii).

This arithmetic criterion allows us to list various examples:

(1) If $2 \in U(k)$, or if 2 is in every maximal ideal of $k$ (e.g. if $k$ has characteristic two), then $Q_{N B}(k)=Q_{F}(k)$. Proof: When $2 \in U(k)$, the equation $x+2 b=1$ can always be solved for $b$; hence $A(k)=k$ and, a fortiori, $A(k)=B(k)$. If 2 is in every maximal ideal, the three conditions $a^{2}+4 c \in U(k)$ for some $c, a+2 b \in U(k)$ for some $b, a \in U(k)$ are all equivalent, by Nakayama's lemma. Thus $A(k)=U(k)=B(k)$.

( 2 ) Consequently, when $k$ is local, we have $Q_{N B}(k)=Q_{F}(k)=Q(k)$, since 2 is either a unit or in the unique maximal ideal. (The same is true for semilocal $k$, see [1, Theorem 4.2.c].)

(3) Let $k=\{(x, y) \in \boldsymbol{Z} \times \boldsymbol{Z} \mid x \equiv y \bmod n\}$ where $2<n \equiv 2 \bmod 4$. Then $(1, n+1)$ is in $B(k)$ but not in $A(k)$, so that $k$ has free quadratic extensions without normal basis. Note that $k$ is connected. This example, with $n=6$, was found (in a different form) by N. Pullman.

A more shocking example is:

(4) Let $k$ be the ring of integers in $Q(\sqrt{D})$ where $D$ is squarefree and $-1>D \equiv 3 \bmod 4$. Then $2+\sqrt{D}$ is in $B(k)$ but not in $A(k)$. Moreover, since $U(k)=\{ \pm 1\}, R(k)=0$. This shows that $0=Q_{N_{B}}(k) \neq$ $Q_{F}(k)$.

(5) If $k$ is quadratically closed (every element is a square) then $Q_{N B}(k)=Q_{F}(k)$. For, suppose $a \in B(k): a^{2}+4 c=u \in U(k)$. Choose $b$ so that $b^{2}=-c$, then $u=(a+2 b)(a-2 b)$, hence $a+2 b \in U(k)$ and $a \in A(k)$.

REMARK. If $2 \in U(k)$, quadratic closure of $k$ implies $Q_{F}(k)=$ $U(k) / U(k)^{2}=0$. If $2 \notin U(k), 0 \neq Q_{F}(k)$ is possible even if $k$ is quadratically closed; for example, $k=\boldsymbol{Z} / 2 \boldsymbol{Z}$. Can this happen with 2 outside some maximal ideal?

(6) Presumably, by a similar argument, $Q_{N B}(k)=Q_{F}(k)$ whenever $k$ is von-Neumann regular. (Of course the only case of interest is when $k$ is not Noetherian and 2 is a zero-divisor lying outside at least one maximal ideal, for if 2 is in every maximal ideal we have the result by Example (1); if 2 is not a zero-divisor it is a unit, and again we have Example (1); and if $k$ is Noetherian it is a finite direct product of fields, and the result follows because $Q, Q_{F}$, and $Q_{N B}$ evidently commute with finite direct products.)

The above results favor bases $\{s, t\}$ with $\operatorname{tr}(s)=0, \operatorname{tr}(t)=1$. A 
different view of the gap between $Q_{N B}$ and $Q_{F}$ is obtained by completing 1 to a basis, as follows:

Lemma 5. If $A$ is a free quadratic extension of $k$ then $1 \in A$ can be completed to a $k$-basis $\{1, d\}$ for $A$, and writing $d^{2}=b_{0}+b_{1} d$ in this basis yields $b_{1}-2 d \in U(A), b_{0}=-N(d)$ and $b_{1}=\operatorname{tr}(d)$. (Here $N(d)=$ $(\sigma d) d$, and $\operatorname{tr}(d)=\sigma d+d$ as above. $)$

Proof. $k \cdot 1$ is a free $k$-direct summand of $A$ by [1, Lemma 1.6]. Let $M$ be a complement: $A=k \cdot 1 \oplus M$. Then $A$ is free if and only if $M$ is free since $M \cong \Lambda_{k}^{2}(A)$. This says that $A$ is free if and only if 1 extends to a basis. Invertibility of $b_{1}-2 d$ follows from $k$-separability of $A$, since $A \cong k[X] /(f(X))$ where $f(X)=X^{2}-\left(b_{0}+b_{1} X\right)$ and $2 d-b_{1}$ is the derivative at $X=d$ of $f(X)$. Finally if $b=\operatorname{tr}(d)$ then $N(d)=(b-d) d=-b_{0}+\left(b-b_{1}\right) d$ gives the rest.

Proposition 6. Let $A$ be a free quadratic extension of $k$ and for each basis of the form $\{1, d\}$ use the lemma to define $x_{d}, y_{d} \in k$ by $(\operatorname{tr}(d)-2 d)\left(x_{d}+y_{d} d\right)=1$. Then the following are equivalent:

(i) $A$ admits a normal basis.

(ii) A admits a basis $\{1, d\}$ with $\operatorname{tr}(d)$ invertible.

(iii) $A$ admits a basis $\{1, d\}$ with $x_{d} \in A(k)$.

Proof. (i) $\Rightarrow$ (ii). If $A=k w \oplus k \sigma(w)$ we have seen that $\operatorname{tr}(w)$ is invertible. $\quad\{1, w\}$ generate $A$ as $k$-module since any element $a w+b(\sigma w)$ can be written as $b(\operatorname{tr} w) \cdot 1+(a-b) w$. It follows that $\{1, w\}$ is a basis, either by checking independence directly using invertibility of $\operatorname{tr}(w)$, or by the general fact that any generating set of $n$ elements for a free (or even just projective) module of rank $n$ is a basis.

(ii) $\Rightarrow$ (iii). The relation $(\operatorname{tr}(d)-2 d)\left(x_{d}+y_{d} d\right)=1$ in $A=k \oplus k d$ implies $\operatorname{tr}(d) x_{d}-2 y_{d} b_{0}=1$ in $k$. If $\operatorname{tr}(d)$ is invertible we can divide this latter equation by it to see that $x_{d}$ is in $A(k)$.

(iii) $\Rightarrow$ (i). Choose $b \in k$ so that $v=x_{d}+2 b \in U(k)$. Put $z=$ $-\left(y_{d} b_{0}+b b_{1}\right) \in k$ (where $d^{2}=b_{0}+b_{1} d$ ) and put $w=z+v d \in A$. Using $\sigma d=b_{1}-d$ and $2 z+v b_{1}=b_{1} x_{d}-2 y_{d} b_{0}=1$ we find $w+\sigma w=1$. Now put $u=v^{-1}, \alpha=-u z$, and $\beta=\alpha+u$ (in k). Then $\beta w+\alpha(\sigma w)=$ $\alpha(w+\sigma w)+u w=\alpha+u z+d=d$. Consequently $\{w, \sigma w\}$ generate $A$ as $k$-module, and therefore form a basis, as before.

2. Comparison with Harrison. In this section we recall (following [2]) the definition of the Harrison cohomology group $H^{2}(k, \Pi)$ and prove directly that it is naturally isomorphic to $R(k)$. As in $\S 1$, $k$ is any commutative ring and $\Pi$ is the group of order two.

Let $\Pi^{i}$ denote the direct product of $i$ copies of $\Pi$ and let $k \Pi^{i}$ 
denote its group-ring. We will construct homomorphisms

$$
U(k \Pi) \stackrel{d^{1}}{\longrightarrow} U\left(k \Pi^{2}\right) \stackrel{d^{2}}{\longrightarrow} U\left(k \Pi^{3}\right),
$$

omit (as is traditional) the verification that $d^{2} d^{1}=0$, and define $H^{2}(k, \Pi)=\operatorname{ker} d^{2} / \operatorname{Im} d^{1}$.

First put $\Delta_{0}(z)=(1, z), \Delta_{1}(z)=(z, z)$, and $\Delta_{2}(z)=(z, 1)$ for $z \in \Pi$, and extend $\Delta_{i}(i=0,1,2)$ to maps $k \Pi \rightarrow k \Pi^{2}$ by linearity. Then, for any $x \in U(k \Pi), d^{1} x=\prod_{i=0}^{2}\left(\Delta_{i} x\right)^{-1^{i}}$. Similarly for $\left(z_{1}, z_{2}\right) \in \Pi^{2}$ define $\Delta_{0}\left(z_{1}, z_{2}\right)=\left(1, z_{1}, z_{2}\right), \Delta_{1}\left(z_{1}, z_{2}\right)=\left(z_{1}, z_{1}, z_{2}\right), \Delta_{2}\left(z_{1}, z_{2}\right)=\left(z_{1}, z_{2}, z_{2}\right)$ and $\Delta_{3}\left(z_{1}, z_{2}\right)$ $=\left(z_{1}, z_{2}, 1\right)$, and extend $\Delta_{i}(i=0,1,2,3)$ to maps $k \Pi^{2} \rightarrow k \Pi^{3}$ by linearity. Then, for any $x \in U\left(k \Pi^{2}\right), d^{2} x=\prod_{i=0}^{3}\left(\Delta_{i} x\right)^{-1^{i}}$. For any $i$ use $\varepsilon$ to denote the augmentation on $k \Pi^{i}$, that is, the ring homomorphism $k \Pi^{i} \rightarrow k$ given by $\varepsilon\left(\sum a_{\sigma} \sigma\right)=\sum a_{\sigma}$ (both sums over $\left.\sigma \in \Pi^{i}\right)$. Some additional notation: $Z(k, \Pi)=\operatorname{ker} d^{2}=$ group of cocycles; $B(k, \Pi)=\operatorname{Im} d^{1}=$ group of coboundaries; and $N G=\operatorname{ker}(\varepsilon: G \rightarrow U(k))=$ subgroup of normalized elements of $G$ (i.e., elements of augmentation 1 ), for any subgroup $G$ of $U\left(k \Pi^{i}\right)$ (for example, $N Z(k, \Pi)=$ normalized cocycles, $N B(k, \Pi)=$ normalized coboundaries).

Proposition 7. Let $\mu=a_{1}+a_{\sigma} \sigma \in U(k \pi),\left(a_{1}, a_{\sigma} \in k\right)$. Then:

(i ) $d^{1} \mu=(\varepsilon(\mu)-x)(1,1)+x(1, \sigma)+x(\sigma, 1)-x(\sigma, \sigma)$ where $x=$ $a_{1} a_{\sigma} / \varepsilon(\mu)$, and

(ii) $\varepsilon\left(d^{1} \mu\right)=\varepsilon(\mu)$.

Proof. (ii) follows from (i). By definition we have $d^{1} \mu=\left(a_{1}(1,1)+\right.$ $\left.a_{\sigma}(1, \sigma)\right)\left(a_{1}(1,1)+a_{\sigma}(\sigma, 1)\right) /\left(a_{1}(1,1)+a_{\sigma}(\sigma, \sigma)\right)$. Letting $\mu^{-1}=b_{1}+b_{\sigma} \sigma$ we have $\varepsilon\left(\mu^{-1}\right)=(\varepsilon(\mu))^{-1}, a_{1} b_{1}+a_{\sigma} b_{\sigma}=1, a_{1} b_{\sigma}+a_{\sigma} b_{1}=0$, and $d^{1} \mu=$ $\left(a_{1}^{2}(1,1)+a_{1} a_{\sigma}(1, \sigma)+a_{\sigma} a_{1}(\sigma, 1)+a_{\sigma}^{2}(\sigma, \sigma)\right)\left(b_{1}(1,1)+b_{\sigma}(\sigma, \sigma)\right)$. Multiplying this out gives $d^{1} \mu=c_{1}(1,1)+c_{2}(1, \sigma)+c_{3}(\sigma, 1)+c_{4}(\sigma, \sigma)$ where $c_{1}=a_{1}^{2} b_{1}+a_{\sigma}^{2} b_{\sigma}, c_{2}=c_{3}=a_{1} a_{\sigma}\left(b_{1}+b_{\sigma}\right)=x$ and $c_{4}=a_{\sigma}^{2} b_{1}+a_{1}^{2} b_{\sigma}$. Since $a_{1}^{2} b_{1}+a_{\sigma}^{2} b_{\sigma}=\left(a_{1} b_{1}+a_{\sigma} b_{\sigma}\right)\left(a_{1}+a_{\sigma}\right)-a_{1} a_{\sigma}\left(b_{1}+b_{\sigma}\right)=\varepsilon(\mu)-x$ and $a_{\sigma}^{2} b_{1}+a_{1}^{2} b_{\sigma}=$ $\left(a_{1}+a_{\sigma}\right)\left(a_{1} b_{\sigma}+a_{\sigma} b_{1}\right)-a_{1} a_{\sigma}\left(b_{1}+b_{\sigma}\right)=-x$, the proof is complete.

Proposition 8. Let $\nu=a_{11}(1,1)+a_{1 \sigma}(1, \sigma)+a_{\sigma 1}(\sigma, 1)+a_{\sigma \sigma}(\sigma, \sigma) \epsilon$ $U\left(k \pi^{2}\right)$. Then:

(i ) $\nu$ is a cocycle $\Leftrightarrow a_{1 \sigma}=a_{\sigma 1}=-a_{\sigma \sigma}$, and

(ii) $\nu$ is a coboundary $\Leftrightarrow \nu$ is a cocycle and $\exists a_{1}, a_{\sigma} \in k$ such that $a_{1}+a_{\sigma} \sigma \in U(k \pi), a_{1 \sigma}=a_{1} a_{\sigma} /\left(a_{1}+a_{\sigma}\right)$ and $a_{11}=a_{1}+a_{\sigma}-a_{1 \sigma}$.

Proof. (ii) is immediate from (i) and part (i) of Proposition 7. For (i), $d^{2}(\nu)$ is by definition $A / B$ where $A$ is the product of $\left(a_{11}(1,1,1)+a_{1 \sigma}(1,1, \sigma)+a_{\sigma 1}(1, \sigma, 1)+a_{\sigma \sigma}(1, \sigma, \sigma)\right)$ and $\left(a_{11}(1,1,1)+\right.$ 
$\left.a_{1 \sigma}(1, \sigma, \sigma)+a_{\sigma 1}(\sigma, 1,1)+a_{\sigma \sigma}(\sigma, \sigma, \sigma)\right)$ and $B$ is the product of $\left(a_{11}(1,1,1)+\right.$ $\left.a_{1 \sigma}(1,1, \sigma)+a_{\sigma 1}(\sigma, \sigma, 1)+a_{\sigma \sigma}(\sigma, \sigma, \sigma)\right)$ and $\left(a_{11}(1,1,1)+a_{1 \sigma}(1, \sigma, 1)+\right.$ $\left.a_{\sigma 1}(\sigma, 1,1)+a_{\sigma \sigma}(\sigma, \sigma, 1)\right)$. Multiplying this out, we see that if $a_{1 \sigma}=$ $a_{\sigma 1}=-a_{\sigma \sigma}$, then each coefficient in $A$ equals the corresponding coefficient in $B$, so that $\nu$ is a cocycle.

The converse is the key point; the proof that follows is implicit in [2]. Let $p_{1}$ (resp. $p_{2}$ ) be the $k$-algebra homomorphism $k \pi^{2} \rightarrow k \pi^{2}$ induced by the map $(x, y) \rightarrow(x, 1)$ (resp. $(x, y) \rightarrow(1, y)$ ) from $\pi^{2}$ to $\pi^{2}$, let $\delta_{1}\left(\operatorname{resp} . \delta_{2}\right)$ be the $k$-algebra homomorphism $k \pi^{3} \rightarrow k \pi^{2}$ induced by the map $(x, y, z) \rightarrow(x, 1)$ (resp. $(x, y, z) \rightarrow(1, z)$ ) from $\pi^{3}$ to $\pi^{2}$, let $\varepsilon: k \pi^{2} \rightarrow k$ be the augmentation and let $j: k \rightarrow k \pi^{2}$ be the inclusion.

Lemma 9. With notation as above, we have the following equalities of maps $k \pi^{2} \rightarrow k \pi^{2}$ :

$$
\begin{aligned}
& \delta_{1} \Delta_{i}=\left\{\begin{array}{lll}
p_{1} & \text { if } & i=1,2,3 \\
j \varepsilon & \text { if } & i=0,
\end{array}\right. \\
& \delta_{2} \Delta_{i}=\left\{\begin{array}{lll}
p_{2} & \text { if } & i=0,1,2 \\
j \varepsilon & \text { if } & i=3 .
\end{array}\right.
\end{aligned}
$$

Proof. Let $\nu=a_{11}(1,1)+a_{1 \sigma}(1, \sigma)+a_{\sigma 1}(\sigma, 1)+a_{\sigma \sigma}(\sigma, \sigma) \in k \pi^{2}$, then $\delta_{1} \Delta_{1}(\nu)^{\prime}=\delta_{1}\left(a_{11}(1,1,1)+a_{1 \sigma}(1,1, \sigma)+a_{\sigma 1}(\sigma, \sigma, 1)+a_{\sigma \sigma}(\sigma, \sigma, \sigma)\right)=a_{11}(1,1)+$ $a_{1 \sigma}(1,1)+a_{\sigma 1}(\sigma, 1)+a_{\sigma \sigma}(\sigma, 1)=p_{1}(\nu) \quad$ and $\quad \delta_{1} \Delta_{0}(\nu)=\delta_{1}\left(a_{11}(1,1,1)+\right.$ $\left.a_{1 \sigma}(1,1, \sigma)+a_{\sigma 1}(1, \sigma, 1)+a_{\sigma \sigma}(1, \sigma, \sigma)\right)=\left(a_{11}+a_{1 \sigma}+a_{\sigma 1}+a_{\sigma \sigma}\right)(1,1)=j \varepsilon(\nu)$, etc.

We can now finish the proof of Proposition 8. If $\nu$ is a cocycle we have $A=B$ where as above $A=\Delta_{0}(\nu) \Delta_{2}(\nu)$ and $B=\Delta_{1}(\nu) \Delta_{3}(\nu)$. Hence $\delta_{1}(A)=\delta_{1}(B)$ and $\delta_{2}(A)=\delta_{2}(B)$. Using the lemma to compute we find $\delta_{1}(A)=\left(\delta_{1} \Delta_{0}(\nu)\right)\left(\delta_{1} A_{2}(\nu)\right)=j \varepsilon(\nu) p_{1}(\nu), \delta_{1}(B)=\left(\delta_{1} A_{1}(\nu)\right)\left(\delta_{1} A_{3}(\nu)\right)=$ $\left(p_{1}(\nu)\right)^{2}, \quad \delta_{2}(A)=\left(\delta_{2} \Delta_{0}(\nu)\right)\left(\delta_{2} \Delta_{2}(\nu)\right)=\left(p_{2}(\nu)\right)^{2}, \quad \delta_{2}(B)=\left(\delta_{2} \Delta_{1}(\nu)\right)\left(\delta_{2} \Delta_{3}(\nu)\right)=$ $p_{2}(\nu)(j \varepsilon(\nu))$. Since $\nu$ is invertible, $p_{1}(\nu)$ and $p_{2}(\nu)$ are also invertible, hence $\delta_{1}(A)=\delta_{1}(B)$ yields $j \varepsilon(\nu)=p_{1}(\nu)$ and $\delta_{2}(A)=\delta_{2}(B)$ yields $j \varepsilon(\nu)=$ $p_{2}(\nu)$. But this means that the three elements $\varepsilon(\nu)(1,1),\left(a_{11}+a_{1 \sigma}\right)(1,1)+$ $\left(a_{\sigma 1}+a_{\sigma \sigma}\right)(\sigma, 1)$ and $\left(a_{11}+a_{\sigma 1}\right)(1,1)+\left(a_{1 \sigma}+a_{\sigma \sigma}\right)(1, \sigma)$ of $k \pi^{2}$ are equal. Hence $a_{\sigma 1}+a_{\sigma \sigma}=0=a_{1 \sigma}+a_{\sigma \sigma}$, and we are done.

Proposition 10. If $\nu=a_{11}(1,1)+a_{1 \sigma}(1, \sigma)+a_{\sigma 1}(\sigma, 1)+a_{\sigma \sigma}(\sigma, \sigma)$ is a cocycle, $a_{1 \sigma} / \varepsilon(\nu)$ is in $k_{4}$.

Proof. We need $1-\left(4 a_{1 \sigma} / \varepsilon(\nu)\right) \in U(k)$, for which it suffices to show $\varepsilon(\nu)-4 a_{1 \sigma} \in U(k)$. Since $\nu$ is a cocycle, $\varepsilon(\nu)-4 a_{1 \sigma}=a_{11}-3 a_{1 \sigma}$. Let $\nu^{-1}=b_{11}(1,1)+b_{1 \sigma}(1, \sigma)+b_{\sigma 1}(\sigma, 1)+b_{\sigma \sigma}(\sigma, \sigma)$. Then, using $1=a_{11} b_{11}+$ $a_{1 \sigma} b_{1 \sigma}+a_{\sigma 1} b_{\sigma 1}+a_{\sigma \sigma} b_{\sigma \sigma}=a_{11} b_{11}+3 a_{1 \sigma} b_{1 \sigma}$ and $0=a_{11} b_{1 \sigma}+a_{1 \sigma} b_{11}+a_{\sigma \sigma} b_{\sigma 1}+$ 
$a_{\sigma 1} b_{\sigma o}=a_{11} b_{1 \sigma}+a_{1 \sigma} b_{11}-2 a_{1 \sigma} b_{1 \sigma}$, we have $\left(a_{11}-3 a_{1 \sigma}\right)\left(b_{11}-3 b_{1 \sigma}\right)=1-3\left(2 a_{1 \sigma} b_{1 \sigma}-\right.$ $\left.a_{1 \sigma} b_{11}-a_{11} b_{1 \sigma}\right)=1$.

Proposition 11. If $x \in k_{4}$ then $\nu=(1-x)(1,1)+x(1, \sigma)+x(\sigma, 1)-$ $x(\sigma, \sigma)$ is a unit in $k \pi^{2}$, and therefore is in $Z(k, \pi)$.

Proof. Let $\nu^{\prime}=(1-3 x)(1,1)-x(1, \sigma)-x(\sigma, 1)+x(\sigma, \sigma)$, then $\nu \nu^{\prime}=(1-4 x)(1,1)$, which is a unit since $x \in k_{4}$, hence $\nu$ is a unit too.

The preceeding propositions show that the rules $\alpha\left(a_{11}(1,1)+\right.$ $\left.a_{\sigma 1}(1, \sigma)+a_{\sigma 1}(\sigma, 1)+a_{\sigma \sigma}(\sigma, \sigma)\right)=a_{1 \sigma} /\left(a_{11}+a_{1 \sigma}\right)$ and $\gamma(x)=(1-x)(1,1)+$ $x(1, \sigma)+x(\sigma, 1)-x(\sigma, \sigma)$ define maps $Z(k, \pi) \rightarrow k_{4}$ and $k_{4} \rightarrow Z(k, \pi)$ respectively. Note that $\alpha \gamma$ is the identity, while $(\gamma \alpha) \nu=\nu / \varepsilon(\nu)$.

Proposition 12. $\gamma$ and $\alpha$ are homomorphisms.

Proof. The computation for $\gamma$ is routine. For $\alpha$, we need $\left(a_{10} c_{11}+\right.$ $\left.a_{11} c_{1 \sigma}+a_{o 1} c_{o \sigma}+a_{\sigma o} c_{\sigma 1}\right) / \varepsilon(\nu) \varepsilon(\mu)=\left(a_{1 \sigma} / \varepsilon(\nu)\right)+\left(c_{1 \sigma} / \varepsilon(\mu)\right)-\left(4 a_{10} c_{1 o} / \varepsilon(\nu) \varepsilon(\mu)\right)$. Putting the right-hand side over the common denominator $\varepsilon(\nu) \varepsilon(\mu)$ and using $\varepsilon(\mu)=c_{11}+c_{1 \sigma}, \varepsilon(\nu)=a_{11}+a_{1 \sigma}, a_{1 \sigma}=-a_{\sigma \sigma}, c_{1 \sigma}=-c_{\sigma \sigma}$ to compute the resulting numerator, we arrive at the left-hand side.

COROLLARY 13. $\alpha$ and $\gamma$ are inverse isomorphisms, $k_{4} \cong N Z(k, \pi)$.

Proof. $\operatorname{Im} \gamma \subseteq N Z(k, \pi)$ and $\gamma \alpha$ is the identity on $N Z(k, \pi)$.

Proposition 14. If $x \in \psi\left(k_{2}\right), \gamma(x) \in N B(k, \pi)$.

Proof. If $x=b(1-b),(1-2 b) \in U(k)$, put $c_{1}=-b /(1-2 b)$ and $c_{\sigma}=(1-b) /(1-2 b)$. Then $\left(c_{1}+c_{\sigma} \sigma\right)(b+(1-b) \sigma)=1$, so $\mu=b+$ $(1-b) \sigma \in U(k \Pi)$, and $d^{1} \mu=\gamma x$.

PROPOSITION 15. If $\nu=a_{11}(1,1)+a_{1 \sigma}(1, \sigma)+a_{\sigma 1}(\sigma, 1)+a_{\sigma o}(\sigma, \sigma)$ is a coboundary then $\alpha(\nu) \in \psi\left(k_{2}\right)$.

Proof. Choose $\mu=\left(a_{1}+a_{o} \sigma\right) \in U(k \pi)$ so that $d^{1} \mu=\nu$. Then $\alpha(\nu)=a_{1 \sigma} / \varepsilon=a_{1} a_{\sigma} / \varepsilon^{2}$ where $\varepsilon=\varepsilon(\mu)=\varepsilon(\nu)$. Now $a_{1} a_{\sigma} / \varepsilon^{2}=\left(a_{1} / \varepsilon\right)\left(1-\left(a_{1} / \varepsilon\right)\right)$, so we have only to check that $1-\left(2 a_{1} / \varepsilon\right) \in U(k)$, or equivalently that $\varepsilon-2 a_{1}=a_{\sigma}-a_{1}$ is a unit. Mimicking the proof of Proposition 10, let $\left(b_{1}+b_{\sigma} \sigma\right)=\mu^{-1}$, then $\left(a_{\sigma}-a_{1}\right)\left(b_{\sigma}-b_{1}\right)=1$.

COROLLARY 16. $\alpha$ and $\gamma$ restrict to inverse isomorphisms $\psi\left(k_{2}\right) \cong$ $N B(k, \pi)$, and they induce inverse isomorphisms $R(k) \cong H^{2}(k, \pi)$. 
Proof. The first statement follows from Propositions 13, 14, and 15. For the second we need, in addition to the definitions, the fact that $Z(k, \pi) / B(k, \pi) \cong N Z(k, \pi) / N B(k, \pi)$. This follows because units of $k$ are always coboundaries: $d^{1} u=u(1,1)$ for any $u \in U(k)$, so that any cocycle $\nu$ represents the same element of $H^{2}(k, \pi)$ as the normalized cocycle $\nu / \varepsilon(\nu)$.

It is worth noting that the proof of Proposition 14 provides an isomorphism between $k_{2}$ and the group of normalized units of $k \pi$ :

Corollary 17. $\lambda(x)=(1-x)+x \sigma$ defines a homomorphism $\lambda: k_{2} \rightarrow U(k \pi)$, and the resulting sequence

$$
0 \longrightarrow k_{2} \stackrel{\lambda}{\longrightarrow} U(k \pi) \stackrel{\varepsilon}{\longrightarrow} U(k) \longrightarrow 1
$$

is split exact.

Proof. The argument which proves Proposition 14 shows that $\lambda$ maps $k_{2}$ to $U(k \pi)$. It is obviously a homomorphism, and the exactness is easily checked.

By definition, $d^{0}$ is the trivial map $U(k) \rightarrow U(k \pi)$, so that $H^{1}(k, \pi)=$ $\operatorname{ker} d^{1} / \operatorname{Im} d^{0}=\operatorname{ker} d^{1}$. The resulting exact sequence can be normalized (i.e., restricted to the augmentation 1 part) to yield the bottom row of a commutative diagram

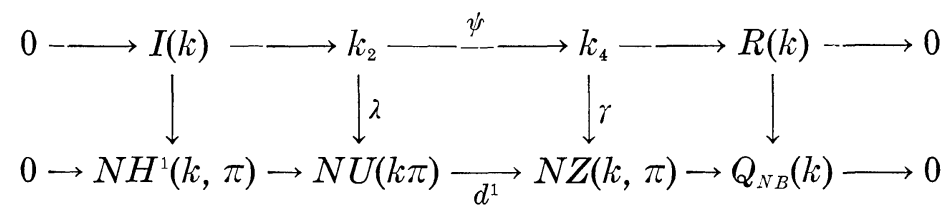

in which the rows are exact and the verticals are all isomorphisms. In fact $N H^{1}(k, \pi)=H^{1}(k, \pi)$ because $d^{1}$ commutes with $\varepsilon$ by Proposition 7 (ii), so we have proved:

COROLLARY 18. $\lambda$ induces an isomorphism $I(k) \rightarrow H^{1}(k, \pi)$, and in particular $k$ is connected $\Leftrightarrow$ the inclusion of $\pi$ in $k \pi$ is an isomorphism $\pi \rightarrow H^{1}(k, \pi)$.

Lifting the description $k_{2} \cong N U(k \pi)$ of normalized units to arbitrary ones yields the following criterion, whose proof is left as an easy exercise:

COROLlaRY 19. Let $\mu=(a+b \sigma) \in k \pi$, then $\mu \in U(k \pi) \Leftrightarrow$ $a^{2}-b^{2} \in U(k)$. 
Finally, it should be pointed out that $\beta \alpha: H^{2}(, \pi) \rightarrow R \rightarrow Q_{N B}$ is the isomorphism of [2], [4]. Thus the cocycle $(\varepsilon(\nu)-x)(1,1)+x(1, \sigma)+$ $x(\sigma, 1)-x(\sigma, \sigma)$ corresponds to the quadratic extension $A=k w \oplus k w^{\prime}$, described by

$$
\left\{\begin{array}{l}
w^{2}=(\varepsilon(\nu)-x) w-x w^{\prime} \\
w w^{\prime}=x w+x w^{\prime}=w^{\prime} w \\
\left(w^{\prime}\right)^{2}=-x w+(\varepsilon(\nu)-x) w^{\prime} \\
w^{\prime}=\sigma w, w=\sigma w^{\prime}
\end{array}\right.
$$

Note that $\left(w+w^{\prime}\right) / \varepsilon(\nu)=1$ in $A$, and consequently $\operatorname{tr}(w)=\varepsilon(\nu)$. Thus the fact, noted in proving Corollary 16, that every cohomology class can be represented by a normalized cocycle, corresponds precisely to the fact (used in proving the converse part of Theorem 3) that any normal basis $\{w, \sigma w\}$ can be replaced by one $\operatorname{with} \operatorname{tr}(w)=1$.

\section{REFERENCES}

1. S. U. Chase, D. K. Harrison, and A. Rosenberg, Galois Theory and Galois Cohomology of Commutative Rings, Amer. Math. Soc., Memoir $\$ 52,1965,15-33$.

2. S. U. Chase and A. Rosenberg, A theorem of Harrison, Kummer theory, and Galois algebras, Nagoya Math. J., 27 (1966), 663-685.

3. A. Micali and O. Villamayor, Sur les Algèbres de Clifford, Ann. Sci. de l'E.N.S. 1 (1968), 271-304.

4. M. (Orzech, A cohomological description of abelian Galois extensions, Trans. Amer. Math. Soc., 137 (1969), 481-499.

5. C. Small, The group of quadratic extensions, J. Pure Appl. Algebra, 2 (1972), 83105 and 395.

Received September 26, 1972.

QUEEN'S UNIVERSITY 



\section{PACIFIC JOURNAL OF MATHEMATICS}

\section{EDITORS}

RICHARD ARENS (Managing Editor)

University of California

Los Angeles, California 90024

\section{R. A. Beaumont \\ University of Washington \\ Seattle, Washington 98105}

\section{J. DugundjI*}

Department of Mathematics University of Southern California Los Angeles, California 90007

D. Gilbarg and J. Milgram

Stanford University

Stanford, California 94305

\section{ASSOCIATE EDITORS}
E. F. BeCKenBaCH
B. H. NeumanN
F. WOLF
K. YoSHIDA

\section{SUPPORTING INSTITUTIONS}

\author{
UNIVERSITY OF BRITISH COLUMBIA \\ CALIFORNIA INSTITUTE OF TECHNOLOGY \\ UNIVERSITY OF CALIFORNIA \\ MONTANA STATE UNIVERSITY \\ UNIVERSITY OF NEVADA \\ NEW MEXICO STATE UNIVERSITY \\ OREGON STATE UNIVERSITY \\ UNIVERSITY OF OREGON \\ OSAKA UNIVERSITY
}

\author{
UNIVERSITY OF SOUTHERN CALIFORNIA \\ STANFORD UNIVERSITY \\ UNIVERSITY OF TOKYO \\ UNIVERSITY OF UTAH \\ WASHINGTON STATE UNIVERSITY \\ UNIVERSITY OF WASHINGTON

$* * *$
$*$
AMERICAN MATHEMATICAL SOCIETY
NAVAL WEAPONS CENTER

The Supporting Institutions listed above contribute to the cost of publication of this Journal, but they are not owners or publishers and have no responsibility for its content or policies.

Mathematical papers intended for publication in the Pacific Journal of Mathematics should be in typed form or offset-reproduced, (not dittoed), double spaced with large margins. Underline Greek letters in red, German in green, and script in blue. The first paragraph or two must be capable of being used separately as a synopsis of the entire paper. Items of the bibliography should not be cited there unless absolutely necessary, in which case they must be identified by author and Journal, rather than by item number. Manuscripts, in duplicate if possible, may be sent to any one of the four editors. Please classify according to the scheme of Math. Rev. Index to Vol. 39. All other communications to the editors should be addressed to the managing editor, or Elaine Barth, University of California, Los Angeles, California, 90024.

100 reprints are provided free for each article, only if page charges have been substantially paid. Additional copies may be obtained at cost in multiples of 50 .

The Pacific Journal of Mathematics is issued monthly as of January 1966. Regular subscription rate: $\$ 60.00$ a year (6 Vols., 12 issues). Special rate: $\$ 30.00$ a year to individual members of supporting institutions.

Subscriptions, orders for back numbers, and changes of address should be sent to Pacific Journal of Mathematics, 103 Highland Boulevard, Berkeley, California, 94708.

PUBLISHED BY PACIFIC JOURNAL OF MATHEMATICS, A NON-PROFIT CORPORATION

Printed at Kokusai Bunken Insatsusha (International Academic Printing Co., Ltd.), 270, 3-chome Totsuka-cho, Shinjuku-ku, Tokyo 160, Japan

* C. R. DePrima California Institute of Technology, Pasadena, CA 91109, will replace J. Dugundji until August 1974. 


\section{Pacific Journal of Mathematics}

Vol. 50, No. $2 \quad$ October, 1974

Mustafa Agah Akcoglu, John Philip Huneke and Hermann Rost, A counter example to the Blum Hanson theorem in general spaces .............

Huzihiro Araki, Some properties of modular conjugation operator of von

Neumann algebras and a non-commutative Radon-Nikodym theorem

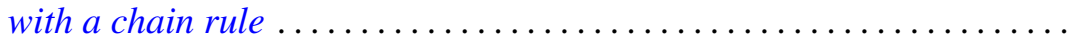

E. F. Beckenbach, Fook H. Eng and Richard Edward Tafel, Global properties of rational and logarithmico-rational minimal surfaces .....

David W. Boyd, A new class of infinite sphere packings ............. 383

K. G. Choo, Whitehead Groups of twisted free associative algebras ........

Charles Kam-Tai Chui and Milton N. Parnes, Limit sets of power series

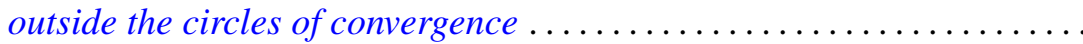

Allan Clark and John Harwood Ewing, The realization of polynomial

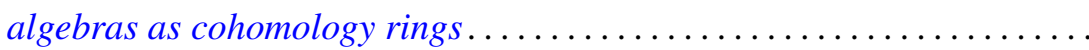

Dennis Garbanati, Classes of circulants over the p-adic and rational

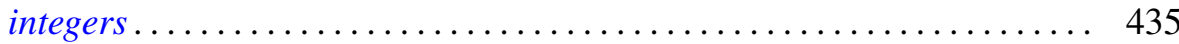

Arjun K. Gupta, On a "square" functional equation ................... 449

David James Hallenbeck and Thomas Harold MacGregor, Subordination and extreme-point theory ............................. 455

Douglas Harris, The local compactness of $v X \ldots \ldots . . . . . . . . . . . .4469$

William Emery Haver, Monotone mappings of a two-disk onto itself which fix the disk's boundary can be canonically approximated by homeomorphisms .................................. 477

Norman Peter Herzberg, On a problem of Hurwitz .................. 485

Chin-Shui Hsu, A class of Abelian groups closed under direct limits and subgroups formation ............................... 495

Bjarni Jónsson and Thomas Paul Whaley, Congruence relations and multiplicity types of algebras.....................

Lowell Duane Loveland, Vertically countable spheres and their wild sets.

Nimrod Megiddo, Kernels of compound games with simple components ....

Russell L. Merris, An identity for matrix functions ........ . .

E. O. Milton, Fourier transforms of odd and even tempered distributions ...

Dix Hayes Pettey, One-one-mappings onto locally connected generalized continua

Mark Bernard Ramras, Orders with finite global dimension

Doron Ravdin, Various types of local homogeneity. .

George Michael Reed, On metrizability of complete Moore spaces ...

Charles Small, Normal bases for quadratic extensions ..

Philip C. Tonne, Polynomials and Hausdorff matrices.... . . 